\title{
Diazepam sedation reduces functional residual capacity and alters the distribution of ventilation in man
}

We measured ventilation and static lung volumes in five fit volunteers in the right lateral decubitus position, while they were fully awake and while sedated with diazepam. We also assessed the distributions of ventilation and perfusion in the lungs, using inhalations and intravenous injections of xenon-127. Diazepam, $0.04 \mathrm{mg} \cdot \mathrm{kg}^{-1}$, was administered every three to five minutes as required to induce and maintain a state of sedation which was moderately heavy. Total doses ranged from 0.16 to $0.38 \mathrm{mg} \cdot \mathrm{kg}^{-j}$. Sedation did not alter minute ventilation, but reduced idal volume, increased breathing frequency and reduced functional residual capacity slightly. Sedation also diminished the normal gradient of ventilation from non-dependent to dependent regions of the lungs. Spontaneous episodes of very small tidal volume breathing during sedation were associated with a marked reduction or cessation of ventilation of the thost depen. dent region of the lungs. There were no sedation-related changes in the distribution of perfusion. These effects of moderately heavy sedation may contribute to the hy. poxaemia and impaiment of pulmonary gas exchange often presen during recovery from general anaesthesia.

\section{Key words}

ANAESTHESIA: recoveTY; SEDATION; LUNG: ventilation, perfusion, static volumes.

From the Departments of Anacsthesia and Nuclear Medicine, University of Western Ontario, London, Canada.

Supported by a Grant-in-Aid from the Ontario Thoracic Society.

Address correspondence to: Dr. R.L. Knill, Department of Anaesthesia, University Hospital, P.O. Box 5339, Postal Station A, London, Ontario, N6A 5A5.
Following inhalational anaesthesia, patients breathing air may be hypoxaemic for periods of time up to three hours, independent of their operative procedure. ${ }^{1-5}$ Factors which can contribute to this early post-anaesthetic hypoxaemia include hypoventilation, nitrous oxide diffusion, ${ }^{6}$ the effects of previous hyperventilation, ${ }^{7}$ atelectasis and/or shivering. ${ }^{8}$ However, these factors are insufficient to explain the phenomenom entirely since, in their absence, hypoxaemia still occurs. $^{2,4}$ This unexplained component of post-anaesthetic hypoxaemia, the result of an increased $\mathrm{P}(\mathrm{A}-\mathrm{a}) \mathrm{O}_{2}$ gradient, is usually mild in young and healthy patients but can be marked in the elderly or in those with preexisting cardiorespiratory disease..$^{5,8}$

Anaesthetizing doses of inhalational anaesthetics usually increase the $\mathrm{P}(\mathrm{A}-\mathrm{a}) \mathrm{O}_{2}$ gradient. Similarly, sedatives sometimes reduce the $\mathrm{PaO}_{2}$ value more than can be accounted for on the basis of a change of $\mathrm{PaCO}_{2} \cdot{ }^{9-11}$ During recovery from inhalational anaesthesia, sedating doses of anaesthetic are present for some time. ${ }^{12}$ Accordingly, we hypothesized that the unexplained component of hypoxaemia during the first hours after anaesthesia is caused by anaesthetic-related sedation present at this time. Since the effect of anaesthesia on the $\mathrm{P}(\mathrm{A}-\mathrm{a}) \mathrm{O}_{2}$ gradient has been related to a reduced functional residual capacity and/or an altered regional distribution of ventilation, ${ }^{13}$ it was the purpose of this study to assess the effects of sedation on these variables. We induced moderately heavy sedation with intravenous diazepam and measured ventilation, static lung volumes and the distributions of ventilation and perfusion. We chose an intravenous rather than an inhaled agent to induce sedation because of difficulties we had encountered in measuring func- 
tional residual capacity in the presence of inhaled agents (see Discussion).

\section{Methods}

This study had been approved by the University of Western Ontario Health Sciences Standing Committee on Human Research.

Five fit volunteers were studied while they were fully awake and then during sedation. Studies were conducted in quiet settings with the subject placed comfortably in the right lateral decubitus position. This position was maintained constant throughout both awake and sedation portions of the study. Variables measured were minute ventilation, static lung volumes (FRC, TLC, RV), regional ventilation and perfusion $\left([\dot{V}]_{r}\right.$ and $\left.[\dot{Q}]_{r}\right)$, and regional ratios of functional residual capacity to total lung capacity $\left(\mathrm{FRC}_{\mathrm{r}} / \mathrm{TLC}_{\mathrm{r}}\right)$. Ventilation and static lung volumes were measured on one occasion and regional variables on another occasion, separated by at least one week. Conditions of study on each occasion were the same.

For the sedation portions of the study, we injected diazepam $0.04 \mathrm{mg} \cdot \mathrm{kg}^{-1}$ intravenously every three to five minutes as necessary to induce and maintain a state of moderately heavy sedation. Injections were made into a free-flowing intravenous infusion. Because of the well-known variability of responses to benzodiazepines among individuals, the end point of diazepam administrations was neither a fixed dose nor a fixed rate of dosing. Rather, the end point was a stable level of moderately intense drowsiness in which the subject lay quietly with his eyes closed but would respond to command and would cooperate in performance of

\begin{tabular}{|c|c|}
\hline \multicolumn{2}{|c|}{ Abbreviations } \\
\hline FRC - & functional residual capacity \\
\hline TLC - & total lung capacity \\
\hline $\mathrm{RV}-$ & residual volume \\
\hline $\mathrm{FRC}_{\mathrm{r}} / \mathrm{TLC}_{\mathrm{r}}$ & $\begin{array}{l}\text { - ratio of regional functional residual } \\
\text { capacity to regional total lung } \\
\text { capacity }\end{array}$ \\
\hline$[\dot{v}]_{r}$ & $\begin{array}{l}\text { - regional ventilation per unit lung } \\
\text { volume at FRC }\end{array}$ \\
\hline$[\dot{Q}]_{r}$ & $\begin{array}{l}\text { - regional perfusion per unit lung } \\
\text { volume at FRC }\end{array}$ \\
\hline
\end{tabular}

the tests. While subjects were sedared, we disturbed them as little as the study protocol allowed. Total cumulative dose of diazepam was limited to $0.4 \mathrm{mg} \cdot \mathrm{kg}^{-1}$.

For all ventilatory measurements, the subject breathed through a Collins large bore mouthpiece with his nostrils occluded tightly by a clip. Functional residual capacity was measured by the helium dilution technique, using a Collins Modular Lung Analyzer fitted with a katharometer. Helium uptake into the blood was assumed to falsely elevate measured FRC values by $1.5 \mathrm{ml} \cdot \mathrm{kg}^{-1}$. Total lung capacity and residual volume were determined from spirometric measurements of inspiratory capacity and expiratory reserve volume, together with the measured values of FRC. Minute ventilation and its subdivisions, tidal volume and breathing frequency, were found from the spirometric record of one minute of resting ventilation taken during the period of helium equilibration. All measurements of ventilation and static lung volumes were made in duplicate and averaged. All measured values were converted to body temperature and ambient pressure, saturated.

Regional ventilation and perfusion were assessed using inhalations and intravenous injections respectively of radioactive xenon. Xenon-127, rather than xenon-133, was selected because it greatly improves resolution of inter-regional differences of xenon concentration in the lungs, due to properties which, coupled with the characteristics of the usual scintillation detector, produce greater scatter rejection. In addition, due to improved penetration, the photons of xenon-127 are more representative of the average xenon concentrations through single slices of the lungs. ${ }^{14}$ During administrations of xenon, a posterior projection of the lungs was obtained with a large field of view scintillation camera interfaced to a Digital Equipment Corporation PDP 11/34 computer.

Regional ventilation per unit lung volume at FRC was determined from an analysis of the wash-in of inhaled xenon- 127 into the lungs while the subject breathed quietly from a closed circuit primed with xenon-127 1.5 millicuries per litre of circuit gas. During wash-in, count rates over each $2 \mathrm{~cm}$ by $2 \mathrm{~cm}$ topographical area of the projected lung fields were recorded as a function of time. An exponential function was fit to the first $1 \frac{1}{2}$ minutes of wash-in data of each area, using least squares technique. 
The exponential parameter so determined is directly proportional to $[\dot{\mathrm{V}}]_{\mathrm{r}}{ }^{15}$

The regional ratio of FRC to TLC was calculated from the measurement of count rates after the inhalation of a known concentration of xenon from FRC to TLC. ${ }^{16,17}$

Regional perfusion per unit lung volume at FRC was cletermined from area count rates observed after the rapid intravenous injection of xenon- 127 dissolved in saline. While the subject held his breath at FRC, xenon-127, one to two millicuries, was injected intravenously via the previously established infusion. After count rates over the lung field stabilized, the subject inhaled slowly to TLC and while he maintained this volume constant, count rates were recorded. From this record, an index of $[Q]_{\mathrm{r}}$ of each region was calculated, using the method of Ball. ${ }^{18,19}$

Count rates present just prior to the start of all xenon manoeuvres were considered background and were subtracted from count rates used to estimate $[\dot{V}]_{r}, F R C_{r} T_{L C}$, and $[\dot{Q}]_{r}$. To derive indices of regional ventilation, FRC/TLC ratios and perfusion from area count rate data, we made conventional assumptions and used conventional calculations. ${ }^{15-19}$ Regional lung variables were expressed as a linear function of vertical distance down the lung ficlds in the lateral position, ${ }^{20}$ using data taken from vertically adjacent topographical areas measuring $2 \mathrm{~cm}$ vertically and $8-10 \mathrm{~cm}$ horizontally and located mid-way between the apices and bases of the lung fields. Individual values of $[\dot{V}]_{r}$ and $[Q]_{r}$ were normalized to an index of 100 , such that had ventilation and perfusion been uniform throughout the lungs, all regional indices would have been 100 .

To test for possible differences in ventilation and global static lung volumes between awake and sedated states, we employed the two tailed Student's t-test for paired data. To test for possible differences in regional lung variables, we used a large sample $\mathrm{Z}$ test for parallelism. ${ }^{21}$

\section{Results}

The subjects were all male. Ranges of ages, heights and weights were respectively $30-37$ years, 164 $193 \mathrm{~cm}$, and $70-100 \mathrm{~kg}$. Periods of sedation ranged from thirty-two to fifty-four minutes. Total doses of diazepam varied from 0.16 to $0.38 \mathrm{mg} \cdot \mathrm{kg}^{-1}$.

While sedated, subjects were quitc drowsy but
TABLE Ventilatory variables

\begin{tabular}{lcc}
\hline & Awake & Sedarion \\
\hline $\begin{array}{l}\text { Minute ventilation } \\
\left(l \cdot \mathrm{min}^{-1}\right)\end{array}$ & $8.4 \pm 0.9$ & $8.6 \pm 1.0$ \\
$\begin{array}{l}\text { Tidal volume } \\
\text { (l) }\end{array}$ & $0.59 \pm 0.12$ & $0.42=0.11^{*}$ \\
$\begin{array}{l}\text { Breathing frequency } \\
\text { Functional residual } \\
\text { capacity } \\
\quad(l)\end{array}$ & $15 \pm 3$ & $21 \pm 4^{*}$ \\
$\begin{array}{l}\text { Total lung capacity } \\
\text { (l) }\end{array}$ & $6.95 \pm 0.57$ & $2.78 \pm 0.57^{*}$ \\
Residual volume & $1.70 \pm 0.38$ & $1.75 \pm 0.48$ \\
(l) & & $6.65 \pm 1.13$ \\
\hline
\end{tabular}

$\mathbf{n}=5$.

All values are means $\pm S$. D

* Significantly different from awake value, $p<0.01$.

always responsive and cooperative. Four of the five subjects werc amncsic for portions of their sedation periods.

Sedation did not alter minute ventilation, but reduced tidal volume and increased breathing frequency ( $p$ values $<0.01$, Table).

Variability of duplicate measurements of FRC was \pm 2.0 per cent or less, TLC \pm 3.9 per cent or less and RV \pm 11.8 per cent or less. Sedation reduced values of FRC slightly $(\mathrm{p}<0.01)$ but had no detectable effect on TLC and RV (Table).

Sedation caused a modest redistribution of ventilation in the lungs, diminishing the gradient of ventilation from non-dependent to dependent regions $(\mathrm{p}<0.01$, Figure 1$)$. In three sedated subjects during wash-in of xenon, episodes of breathing with tidal volumes less than half usual awake values occurred. Associated with these episodes in all three subjects was a further redistribution of ventilation in the lungs, characterized by a marked reduction or cessation of ventilation of the most dependent region (e.g. Figure 2).

Sedation did not detectably alter $\mathrm{FRC}_{\mathrm{r}} / \mathrm{TLC}_{\mathrm{r}}$ ratios nor regional perfusion.

\section{Discussion}

The level of sedation we studied was intended to be moderately heavy, akin to what is often present in patients during early recovery from inhalational anaesthesia. We used diazepam rather than an inhalational anaesthetic to produce this state because we had found that inhaled agents interfere 


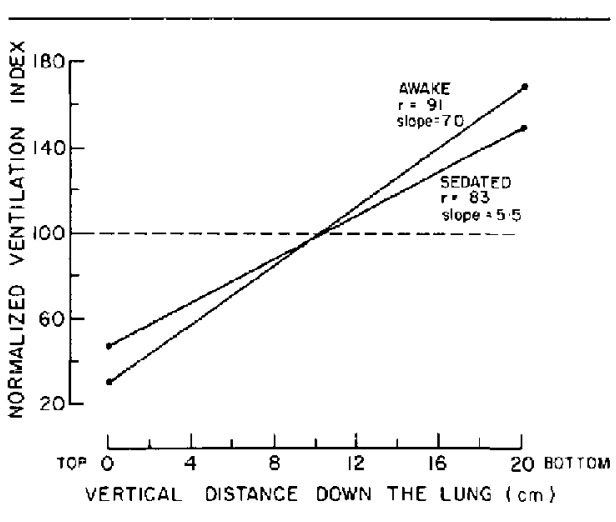

FIGURE I Mean regional yentilation normalized to an index of 100 and represented as a linear function of vertical distance down the lung fields. In the awake state, there was a normal gradient of yentilation from non-dependent to dependent regions of the lungs. Diazepam sedation reduced this gradient slightly $(\mathrm{p}<0.01)$.

with measurements of helium concentration by our katharometer* in complex and variable ways. Since we were interested in the effects of a state rather than a particular dose of diazepam, we individualized diazepam administrations to induce and maintain what was judged to be a similar intensity of sedation in all subjects. To minimize disturbance of the sedated state, we studied subjects in quiet settings and avoided conversing with them and stimulating them as much as was possible within the study protocol. $\dagger$

Methods used to measure ventilation and static lung volumes were standard. To estimate regional ventilation and pcrfusion, we employed an isotope of xenon and a type of scintillation detector that together provide better than usual resolutions of inter-regional differences in these variables ${ }^{14} \mathrm{We}$ studied subjects in the lateral decubitus position on the assumption that this position would enhance the

*Employed in the determination of FRC by helium dilution.

HIt should be noted that measurements of TLC, RV, $F_{R C} T_{L} C_{r}$ and $\left[\dot{Q}_{\mathrm{r}}\right.$ required that subjects perform ventilatory manoeuvres (maximal inhalations and exhalations, and breath holding). We cannot exclude the possibility that performance of these manoeuvres altered the sedated state and accordingly, effects sedation may have otherwise had. detectability of any gravity-related changes in regional ventilation and perfusion.

Moderately heavy sedation had three effects which were observed consistently in all subjects: (1) it reduced tidal volume and increased breathing frequency, without detectably altering minute ventilation; (2) it reduced functional residual capacity, without delectably affecting total lung capacity or residual volumc; and (3) it altered regional ventilation, without detectably affecting regional perfusion.

When sedatives, hypnotics and anaesthetics depress ventilation in humans, they also reduce tidal volume and increase breathing frequency. Although diazepam sedation did not alter minute ventilation in this study, it reduced tidal volume and increased breathing frequency (Table). This independent effect on breathing pattern has been observed previously with diazepam sedation, ${ }^{22}$ as well as with diethyl ether anaesthesia. ${ }^{23}$ Perhaps the ventilatory control systems which subdivide ventilation into tidal volume and breathing frequency are more susceptible to pharmacologically induced disturbance than are the systems which regulate total ventilation.

The reduction of functional residual capacity observed with sedation in this study was small, averaging about six per cent (Table). It is possible that this reduction was factitious, i.e., due to falsely high FRC values in the awake state related to subject anxiety. ${ }^{24}$ However, determinations of FRC in this study were highly reproducible, and an anxiety relieving dose of morphine $\left(0.2 \mathrm{mg} \cdot \mathrm{kg}^{-3}\right.$ intramuscularly) did not reduce FRC values in twelve pre-operative patients studied by one of us previously.*

Other sedatives and anaesthetics administered in sedating doses have also been reported to reduce FRC. Intramuscular hydroxyzine $1 \mathrm{mg} \cdot \mathrm{kg}^{-1}$ or droperidol $0.07 \mathrm{mg} \cdot \mathrm{kg}^{-1}$ decreased FRC one to two hours after administration ${ }^{25}$ and halothane $0.2 \mathrm{MAC}$ reduced FRC in three of four subjects. ${ }^{26}$ However, in a recent careful study, sedation with halothane or enflurane did not consistently reduce FRC values mcasured with a body plethysmograph and, in some cases, sedation actually increased FRC. ${ }^{27}$ The explanation for these different results is not clear. Perhaps sedated subjects of the latter

*Baxter B., Knill R.L. unpublished observations. 


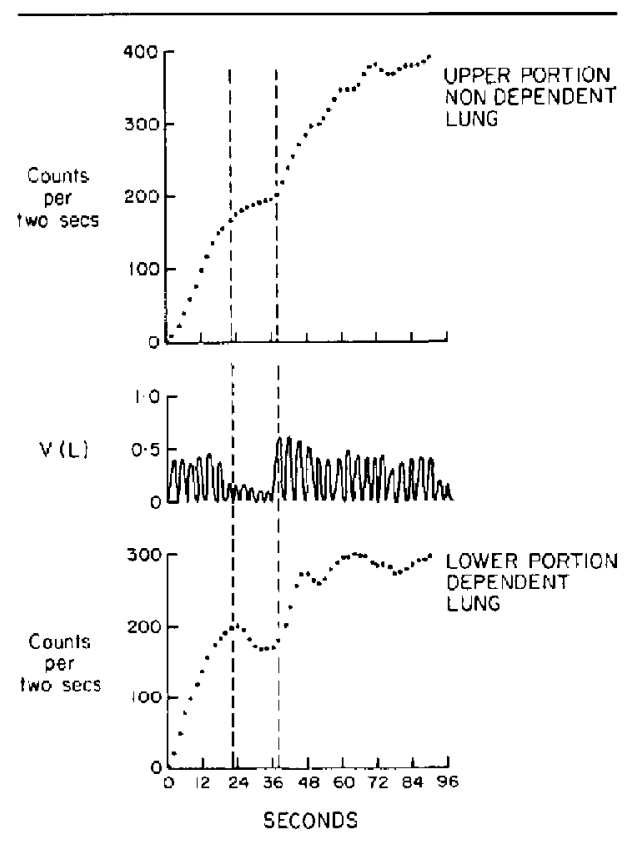

FIGURE 2 Regional ventilation assessed by wash-in of xeлon-127 in a sedated subject, showing the effects of a spontaneous episode of very small tidal volume breathing. Depicted are count rates as a function of time observed over the upper zone of the non-dependent lung and the lower zone of the dependent lung, in relation to the spirometric trace of tidal volume. During the initial period of wash-in, when tidal volumes were 0.4 to $0.5 \mathrm{l}$, count rates increased slightly more quickly in the dependent zone compared to the non-dependent, indicating a greater proportion of ventilation in the dependent region. From 22 to 38 seconds, tidal volumes decreased spontaneously to less than $0.3 \mathrm{I}$. Coinciding with this period, wash-in of xenon in non-dependent zones decreased slightly, but wash-in in the most dependent zone ceased entircly indicating a redistribution of ventilation such that there was little or no ventilation of the most dependent rcgion. At 38 seconds, larger tidal volumes resumed and, with them, the original patterm of wash-in of xenon.

study were more stimulated (and thus less sedated) than subjects of the other quoted studies since, unlike the others, they were confined to an enclosed box and had an oesophageal tube in place for the duration of the study. Alternatively, if sedation were to cause trapping, it could reduce the volume of gas at FRC found by an airway equilibration technique without reducing total thoracic gas volume at FRC determined with a body plethysmograph.
The magnitude of FRC reduction associated with sedation in this study was somewhat less than that usually observed with inhalational or intravenous anaesthesia, although the reported effects of anaesthesia vary considerably. ${ }^{13}$ In work which preceded this study, we noted that less sedation produced by smaller doses of diazepam given to three of the subjects of the present study had no detectable effect on FRC; similarly, Cottrell et al. observed that a relatively small dose of diazepam administered intramuscularly $\left(0.1 \mathrm{mg} \cdot \mathrm{kg}^{-1}\right)$ did not alter FRC values. ${ }^{25}$ Together, these observations suggest that the reduction of FRC associated with sedatives and anaesthetics is dose-related and that the threshold dose for a detectable effect is one which produces a moderately intense level of sedation.

The observed effect of diazepam sedation on FRC was associated with no detectable change in either total lung capacity or residual volume (Table). After reviewing studies of anaesthetic effects on the lungs and the chest wall, Rehder $e t a l$. concluded that the reduced FRC associated with anaesthesia was probably due to an action of anaesthetics on the mechanical properties of the chest wall. ${ }^{13}$ One of us observed previously that halothane sedation reduced the tonic activity of both diaphragm and intercostal muscles. ${ }^{26} \mathrm{~A}$ reduction of tonic inspiratory muscle activity would explain the reduced FRC occurring independent of change in TLC and RV, since inspiratory muscle activity has its greatest effect on lung volume at and about FRC, due to the slope of the pressure-volume relationship of the lungs and chest wall at and about that volume.

Diazepam sedation altered the regional distribution of ventilation assessed by wash-in of inhaled xenon. Sedation diminished the normal gradient of ventilation from non-dependent to dependent regions of the lungs or, in other words, caused ventilation to be distributed more uniformly throughout the lungs (Figure 1). The change, although modest, was observed consistently in our subjects and was qualitatively similar to that reported for inhalational anaesthesia with spontaneous breathing. ${ }^{28,29}$

Of particular interest was the chance observation that when tidal volumes became quite small during sedation, there was a further redistribution of ventilation. Coinciding with periods of small tidal volume breathing that occurred spontaneously during inhalations of xenon in three sedated subjects, 
wash-in of xenon in the most dependent zone of the lungs ceased entirely while wash-in in nondependent zones declined only slightly (Figure 2). These data indicate a marked reduction or cessation of ventilation in the dependent region of the lungs while ventilation continued elsewhere - in other words, a considerable redistribution of ventilation away from the dependent region.* $\mathrm{A}$ qualitative relationship to tidal volume was clear and, to our knowledge, has not been observed previously in spontaneously breathing subjects - awake, sedated or anaesthetized.

The basis for the altered regional ventilation we observed during sedation is unclear. Classic studies of the distribution of xenon boli inhaled from various lung volumes by awake subjects predict that spontaneous inspirations from a reduced FRC would redistribute ventilation away from the dependent zones of the lungs. ${ }^{30}$ This would be particularly so if FRC were close to or below closing capacity and/or if the vertical gradient of expansion of the lung at FRC were increased. In this study, we observed that diazepam sedation reduced FRC values, but only slightly. We did not measure closing capacity and we detected no alteration in the vertical gradient of lung expansion at FRC, as assessed by FRC $_{\mathrm{r}}$ TLC $_{\mathrm{r}}$ ratios. $\dagger$ We did observe a relationship between the distribution of ventilation and tidal volume when tidal volumes were quite small (Figure 2), suggesting that, in some circumstances at least, tidal volume may be important in

\footnotetext{
*There was not only cessation of xenon wash-in in the dependent zone but, subsequently, a reduction of xenon concentration there (Figure 2). The abrupt cessation of wash-in in the dependent zone, coinciding exactly with the onset of small tidal volume breathing, is strong evidence of either a marked reduction or at cessation of ventilation of that region with fresh gas. The subsequent reduction of local xenon concentration may have been due $t o$, in addition, clearance of xenon by the pulmonary circulation and/or cross ventilation of that region with regions having lower xenon concentrations. †The absence of detectable change in regional FRC/TLC ratios is not in keeping with our observations of a reduced global FRC and unchanged global TLC. This apparent inconsistency may have been due to the lesser accuracy of measurements of FRC $_{r} / T_{L C} C_{T}$ and/or to the effort dependency and thus variability of measurements of TLC.
}

the effect. However, the relative roles of tidal volume and other potential factors remains to be determined.

Diazepam sedation did not alter regional distribution of perfusion assessed by the intravenous xenon technique. Anaesthesia also does not affect regional perfusion assessed in this way. ${ }^{13} \mathrm{~A}$ reduction of the gradient of ventilation from non-dependent to dependent lung without adjustment in regional perfusion would be expected to impair intrapulmonary gas exchange - and especially if ventilation were to cease in the dependent regions. Unfortunately, we did not measure arterial blood gas values in this study. In other studies in which sedation was probably less intense, sedation reduced $\mathrm{PaO}_{2}$ values more than can be accounted for on the basis of small increases in $\mathrm{PaCO}_{2}$. $9-11$

In summary, a state of moderately heavy diazepam sedation in fit subjects in the right lateral decubitus position reduced tidal volume, increased breathing frequency, reduced FRC slightly and diminished the normal gradient of ventilation from non-dependent to dependent regions of the lungs, especially when tidal volumes were quite small. These findings cannot necessarily be extrapolated to patients receiving therapeutic sedation nor to patients recovering from inhalational anaesthesia, where drugs and body position may be different and other variables present. Nonetheless, these observations indicate that important respiratory effects of anaesthesia can extend to sub-anaesthetic or sedating doses of an anaesthetic agent and they lend support to the hypothesis that anaesthetic-related sedation contributes to the hypoxaemia and impairment of pulmonary gas exchange often present in patients recovering from anaesthesia.

\section{Acknowledgements}

The authors wish to acknowledge the technical assistance of $\mathrm{K}$. Wilkins and $\mathrm{J}$. Clement and the secretarial assistance of $\mathrm{L}$. Nolan and J. Traher. We also wish to thank K. Campbell for statistical advice, Dr. L. Reese for the use of the Nuclear Medicine facility at St. Joseph's Hospital, London, Ontario and Dr. J. Roberts for helpful criticisms of the manuscript. 


\section{References}

1 Nunn JF, Payne JP. Hypoxaemia after general anaesthesia. Lancet 1962; 2: 631-2.

2 Marshall $B E$, Millar RA. Some factors inluencing post-operative hypoxaemia. Anaesthesia 1965; 20: $408-28$

3. Thompson DS, Eason CN Hypoxaemia immediately after operation. Am J Surg 1970; 120: 649-51.

4 Marshall BE, Cohen PJ, Klingenmaier $C H, O u k$ berg $S$. Pulmonary venous admixture before, during and after halothane: oxygen anaesthesia in man. J Appl Physiol 1969; 27: 653-7.

5 Kiamura H, Sawa $T$, Ikezono E. Post-operative hypoxemia: the contribution of age to maldistribution of: ventilation. Anesthesiology 1972; 36: 244-52.

6 Frumin $M J$, Edelist $G$. Diffusion anoxia: A critical reappraisal. Anesthesiology 1969; 31: 243-9.

7 Salvatore Ad, Sullivan SF, Papper, EM Pastoperative hypoventilation and hypoxemia in man after hyperventilation. N Engl J Med 1969; 280 467-70.

8 Bay J, Nunn JF, Prys-Roberts C. Factors influencing arterial $\mathrm{PO}_{2}$ during recovery from anaesthesia. BrJ Anaesth 1968; 40: 398-407.

9 Dalen JE, Evans GL, Banas JS, Brooks $H L$, Paraskos $J A$, Dexier $L$. The hemodynamic and respiratory effects of diazepam (Valium). Anesthesiolory 1969; 30: $259-63$.

10 Kopman EA, Ramierez-Inawat RC. Arterial hypoxaemia following premedication in patients with coronary artery disease. Can Anaesth Soc J 1980; 27: $132-4$.

11 Pierce JA, Garofalo ML. Prc-opcrative medica tion and its effect on blood gases. JAMA 1965; 194; 99-102.

12 Gelb AW, Knill RL. Sub-anaesthetic halothane: Its effect on regulation of ventilation and relevance to the recovery room. Can Anaesth Soc J 1978; 25: 488-94.

13 Rehder $K$, Sessler AD, Marsh HM. General anaesthesia and the lung. Am Rev Respir Dis 1975; 112: $541-63$.

14 Coates $G$, Nahmias $C$. Xenon-127, a comparison with xenon-133 for ventilation studies. J Nucl Med 1977; 18: 221-5.

15 Prato FS, Kurdyak R, Saibil E, Rider WD, Aspin $N$. Regional and total lung function in patients following pulmonary irradiation. Investigative Radiology $1977 ; 12: 224-37$.

16 Milic-Emili J, Henderson JAM, Dolovich MD, Trop
$D$, Kaneko $K$. Regional distribution of inspired gas in the lung. J, Appl Physiol 1966; 21: 749-59.

17 Bigler $A H$, Renzetti $A D$ Jr. Regional lung expansion using ${ }^{133} \mathrm{Xe}$ : Theory and methods. J Appl Physiol 1973; 35: 770-1.

18 Ball WC, Stewart PB, Newsham LGS, Bates DV Regional pulmonary function studies with xenon133. J Clin lnvest 1962; 41: 519-31.

19 Anthonisen NR, Milic-Emili J. Distribution of pulmonary perfusion in erect man. J Appl Physiol 1966; $21: 760-6$.

20 Rehder $K$, Sessler $A D$. Rodarte JR. Regional intrapulmonary gas distribution in awake and anaesthetized - paralyzed man. J Appl Physiol 1977; 42: 391-402.

21 Kleinbaum DG. Applied Regression Analysis and Other Multivariable Methods, pp. 95-105. The University of North Carolina at Chapel Hill, Duxbury Press North Scituate, Massachusets, 1978.

22 Carchlove RFH, Krafer ER. The effects of diazepam on the ventilatory response to carbon dioxide and on steady-state gas exchange. Anesthesiology 1971; 34: 9-13.

23 Larson CP Jr, Eger El, Muallem M. Buechel DR, Munson ES, Eisele $J H$. The effects of diethyl ether and methoxyflurane on ventilation: II. A Comparative study in man. Anesthesiology 1969; 30: 17484.

24 Goldman MD, Morris TH, Kendall LG, Loh L. The effects of fentanyl and thiopental on breathing in man. Advances in Experimental Medicine and Biology 1978; 99: 117-22.

25 Cottrell JE, Wolfson $B$, Siker ES. Changes in airway resistance following droperidol, hydroxyzine and diazepam in normal volunteers. Anesth Analg 1976; 55: 18-21.

26 Muller N, Bryan AC, Lam A, Knill RL. Importance of respiratory muscle tonic activity in the maintenance of the functional residual capacity (FRC). Fed Proc 1979; 38: 1382.

27 Gelb A, Rehder $K$. The effect of halothane and enflurane sedation on pulmonary mechanics in man. Fed Proc 1981; 40: 541 .

28 Rehder $K$, Sessler $A D$. Function of each lung in spontaneously breathing man anesthetized with thiopental-mcperidine. Anesthesiology 1973; 38: $320-327$.

29 Couture J, Picken J, Trop D, et al. Airway closure in normal, obese and anaesthetized supine subjects. Fed Proc 1970; 29: 269 
30 Kaneko K, Milic-Emili J, Dolovich MB, Dawson A, Bates, $D V$. Regional distribution of ventilation and perfusion as a function of body position. $J$ Appl Physiol 1966; 21: 767-774.

\section{Résumé}

Nous avons mesuré la ventilation et les volumes pulmonaires statiques chez cinq (5) volontaires sains en décubitus latéral droit alors qu' ils étaient éveillés ou sous l'effer d'une sédation au diazépam. Nous avons aussi déterminé la distribution de la ventilation et de la perfusion dans les poumons en utilisant du xénon-127 en inhalation et en injection intraveineuse. Du diazépam, $0.04 \mathrm{mg} \cdot \mathrm{kg}^{-1}$ a été administré a intervalles de trois à cinqu minutes de façon à induire et à maintenir un état de sédation de profondeur moyenne. Les doses totales s'échelonnaient de 0.16 à $0.38 \mathrm{mg} \cdot \mathrm{kg}^{-1}$. Cette sédation n'a pas produit de changement de la ventilation minute mais par contre a provoqué une réduction du volume courant, une augmentation de la fréquence respiratoire et une diminution légère de la capacité résiduelle fonctionnelle. La sédation a aussi altéré la distribution normale de la ventilation dans le poumon. Des épisodes spontanés de respiration à perit volume courant durant la sédation on accompagné une réduction marquée ou un arrêt total de la venilation dans les régions les plus déclives du poumon. Il n'y a pas eu de changement de la distribution de la perfusion en association avec la sédation. Ces effets d' une sédation modérée au diazépam peuvent contribuer à l'hypoxémie et aux problèmes d'échanges gazeux pulmonaires souvent présents durant la période de recouvrance d' une anesthésie générale. 